\title{
A study of microemboli monitoring of atherosclerotic thrombotic cerebral infarction and artery stenosis
}

D.J. Sun ${ }^{1}$, A.X. Zhuang ${ }^{1}$, Q.H. Zeng' ${ }^{1}$ Y.L. Jiang ${ }^{1}$, J.D. Jiang ${ }^{1}$, S.Q. Feng ${ }^{2}$, Y. Zhang ${ }^{3}$, H.M. Huang ${ }^{4}$, H.X. Nie ${ }^{1}$ and L. Liu ${ }^{1}$

'Department of Neurology, LianyunGang Second People's Hospital (Bengbu Medical College, Lianyungang Hospital), District Xinpu, LianyunGang, China

${ }^{2}$ Neurological Examination Division, LianyunGang Second People's Hospital (Bengbu Medical College, Lianyungang Hospital), District Xinpu, LianyunGang, China ${ }^{3}$ Department of Radiology, LianyunGang Second People's Hospital (Bengbu Medical College, Lianyungang Hospital), District Xinpu, LianyunGang, China

${ }^{4}$ Department of Ultrasound, LianyunGang Second People's Hospital (Bengbu Medical College, Lianyungang Hospital), District Xinpu, LianyunGang, China

Corresponding author: J.D.Jiang

E-mail: jiandongjiang@yeah.net

Genet. Mol. Res. 13 (3): 6734-6745 (2014)

Received May 16, 2013

Accepted October 30, 2013

Published August 28, 2014

DOI http://dx.doi.org/10.4238/2014.August.28.17

ABSTRACT. This study aimed to assess the relationship between the recurrence and prognosis of patients with acute middle cerebral artery infarction, atherosclerotic brain infarction, and the existence of microemboli. We continuously enrolled patients with acute atherosclerotic thrombotic cerebral infarction artery stenosis. We performed transcranial Doppler color ultrasound micro emboli monitoring, color Doppler ultrasound carotid artery tests, intracranial 
and carotid artery magnetic resonance angiography, impairment evaluation of nerve function, and registration of stroke recurrence and stroke mortality. Of the 49 patients enrolled in the study, 123 main arteries presented atherosclerotic stenosis or formed plaques, and 33 patients had symptomatic stenosis. Patients with symptomatic stenosis have a higher incidence of microemboli than patients with asymptomatic stenosis $(\mathrm{P}=0.009)$. The microembolus-positive rate increased in patients with unstable plaques $(\mathrm{P}=0.001)$. Patients who were microembolus-negative were more likely to show a neural function deficient NIHSS (National Institutes of Stroke Scale) score improvement than patients who were microembolus-positive at one week $(\mathrm{P}=0.026)$. However, we found no significant difference between $\mathrm{mRS}$ (modified rankin scale) score $(\mathrm{P}=0.319)$, relapse, and death $(\mathrm{P}=0.179)$. The rate of microembolus-positivity increased in patients with atherosclerotic thrombotic cerebral infarction and unstable plaques. Patients who were microembolus-negative were more likely to show an improvement of neural function deficiency than patients with microembolus-positivity at one week $(\mathrm{P}=0.026)$.

Key words: Atherosclerotic thrombotic cerebral infarction; Microemboli; Color trans-cranial Doppler ultrasound; Stroke recurrence

\section{INTRODUCTION}

Spencer et al. (1990) initially monitored cerebral blood flow in patients treated with carotid endarterectomy with transcranial Doppler ultrasound (TCD) and found abnormal signals of solid particles, which were named microembolic signals (MES). Subsequently, the journal Stroke published expert consensus of the diagnostic criteria of microembolus signals (Ackerstaff et al., 1995). Since then, TCD microemboli are often used in cerebral angiography, carotid artery stenting, carotid endarterectomy, and as monitoring indicators preventing thrombotic events in surgery. Studies have shown that the presence of microemboli is related to the lesion numbers shown by MRI (magnetic resonance imaging) and DWI (diffusion-weighted imaging) and the lesion characteristics in the skull of patients with cerebral infarction (Kimura et al., 2001; Wong et al., 2002; Nakajima et al., 2007). The recurrence probability of cerebral infarction increased in microembolus-positive patients (Valton et al., 1998; Markus et al., 2005; Nakajima et al., 2007; Iguchi et al., 2007). Recent studies also found that the presence of microemboli is associated with the severity of carotid stenosis (Markus et al., 2005; Ritter, 2008). However, studies of microemboli are mostly focused on carotid atherosclerosis and cerebral infarction with cross-sectional study, with little concern regarding intracranial atherosclerotic cerebral infarction. We performed angiography to test patients for atherosclerotic cerebral infarction and executed dynamic tests of microemboli $48 \mathrm{~h}, 1$ week, and 3 months after the disease onset. We also explored the incidence of microemboli in patients with artery stenosis and cerebral infarction, and the effects of microemboli on neurological functional deficits after stroke, stroke recurrence, and death. 


\section{MATERIAL AND METHODS}

\section{Data}

We continuously enrolled patients hospitalized for acute cerebral infarction who had artery atherosclerosis and received microembolus monitoring, and whose relevant information was recorded. Inclusion criteria included: 1) cerebral infarction patients with atherosclerosis (arterial atherosclerotic cerebral infarction was diagnosed according to the 2002 modified version of the Trial of ORG 10172 in Acute Stroke Treatment (TOAST) classification (Goldstein et al., 2001); 2) a patient aged between 18 and 80 years old; 3) cerebral infarction within $48 \mathrm{~h}$; and 4 ) the cooperation of the patients or their relatives, and signed informed consent. We referenced the study of DeGraba et al. (1999) to classify patients whose neurologic impairment NIHSS score was 0-6 into the mild to moderate group, while patients whose NIHSS score was more than 6 were classified into the severe group. Exclusion criteria included the following: 1) the temporal ultrasound windows of the head were closed; 2) the patient's condition was serious, and they could not tolerate $30 \mathrm{~min}$ of microembolus monitoring and vascular ultrasound of the neck; and 3) patients who refused to cooperate.

\section{TCD microemboli monitoring}

It has been previously established that all enrolled patients admitted to hospital should receive microembolus detection as soon as possible within $48 \mathrm{~h}$ (Hao et al., 2010). Patients with positive microembolic signals would undergo microemboli detection again after one week. Continuously positive patients should undergo a third detection. Microemboli monitoring required the Embodop transcranial Doppler ultrasound detector (DWL Company, Germany). All patients were required to close their eyes and sit on the examining table quietly. First, 2 or $4 \mathrm{MHz}$ probes were used to process the routine test of intracranial and extracranial blood vessels. Then, we fixed the probe on the lesion side of the temple window and cerebral middle artery, and processed the microembolus monitoring in the initial segment of the middle cerebral artery or distal microstenosis. The sampling depth was $48-58 \mathrm{~mm}$, the distance between two points was $\geq 6 \mathrm{~cm}$, the sampling volume was $6-12 \mathrm{~cm}$, and the relative intensity threshold of microemboli was $>5 \mathrm{~dB}$, as far as possible to reduce the gain to get a clear spectrum. The microembolus monitoring time of each patient was 30 minutes. Each case needed to be offline to play back the signal after the monitoring was completed in order to exclude pseudo-difference and interference. The Kay Professional TCD software VI 3.3 (Germany) was used to record the results. Microembolus monitoring was operated by the same professionals certified who had received systematic training, and the results were analyzed by a experienced radiologist from the Department of Neurology. We used the 1995' standards to identify the microemboli for which (Ackerstaff et al., 1995): 1) the duration was short $(<300$ $\mathrm{ms}) ; 2$ ) the intensity was greater than the background blood flow signal ( $\geq 3 \mathrm{~dB}) ; 3$ ) unidirectional signal; 4) the audio signal was expressed as a "crackling sound" or "birdsong"; 5) the audio signal appeared random in the cardiac cycle; and 6) a time delay occurred between the two monitoring depths. The methods to design a pseudo-differential are: a wide pseudo-differential spectrum that was biphasic, or even had bilateral symmetry, in which the high intensity portion is in the low frequency region of the signal. 


\section{Carotid artery ultrasound detection}

This study was designed to use the HD ISonoCT25000 color Doppler ultrasonic diagnostic apparatus (Philips, USA), the probe frequency was 5-12 MHz. Patients rested in a supine position, with a neck placed in a pillow, the head thrown back as far as possible, and turned to check the opposite side. Detection from the root segment of the common carotid artery in turn showed the common carotid artery, bifurcation, and the extra-cranial part of the carotid artery until the unclear artery into the skull. The probe was continuously scanned from the longitudinal and cross-sectional cut. A two-dimensional ultrasound was used to observe the arterial wall structure, echo, and lesions involvement, to measure the diameter of vessels and medial thickness, and to record the location, size, surface morphology, internal structure, and echo characteristics of the plaque. We observed the situation of blood filling and collected the blood spectrum to measure the flow rate. The angle between the beam and the direction of blood flow in the sampling should be less than $60^{\circ}$. Carotid ultrasound criteria included that the thickness of carotid intima-media $>1.0 \mathrm{~mm}$ was diagnosed as thickening, while $>1.2 \mathrm{~mm}$ was diagnosed as atherosclerotic plaque formation. Plaques were divided into the following four kinds: 1) uniform low-echo lipid soft plaque; 2) echo fibrous plaques; 3) hyperechoic or calcifiedhard plaque with acoustic shadow; and 4) ulcer-type plaques with internal uneven echo, irregular surface morphology, and incomplete fibrous cap. The results of the carotid ultrasound can be simplified into three: 1) no plaque; 2) stable plaque, including fast fibrous plaque and calcified hard plaque; and 3) unstable plaque, including soft plaque, ulcerative mixed plaques.

\section{Head MRI examinations}

All enrolled patients received head MRI DWI detection. Those whose clinical manifestations appeared as severe progression or recurrence should review cranial MRI DWI. All patients received intracranial and neck aorta MRA examination.

If any of the TCD results of the head and neck artery, carotid ultrasound, and MRA imaging examination were in accordance with the criterion of diagnosis of vascular stenosis, the patient can be defined as having vessel stenosis (Hao et al., 2010).

\section{Nerve function defect evaluation}

The patients underwent nerve function impairment NIHSS grading immediately after admission and after one week, and received an mRS score when they left hospital and were followed up to check the incidence at three months later and registered the situation of stroke recurrence and stroke death.

\section{Stroke risk factors and general observation indicators}

We recorded common cerebrovascular disease risk factors and general information, such as the patient's age, gender, smoking history, drinking history, history of hypertension, diabetes mellitus, history of stroke, coronary heart disease, history of atrial fibrillation, blood glucose, blood lipids, blood homo-cysteine, C-reactive protein, fiber fibrinogen, and glutamyl trans-peptidase levels. 


\section{Statistical methods}

We used the SPSS11.5 software package (Chicago, IL, USA) for statistical analysis. We used the chi-square test for the count data, the Student $t$-test to measure differences between the two groups, and single factor analysis of variance (ANOVA) for multiple groups comparisons. Microembolus-related factors were analyzed by logistic regression. $\mathrm{P}<0.05$ was considered to represent statistically significant differences for two-sided tests.

\section{RESULTS}

\section{Study cohort}

From January 2008 to October 2011, we collected data from nearly 1000 cases with ischemic stroke from the Department of Neurology at the 2nd People's Hospital in Lianyun Gang (Lianyun Gang-Affiliated Hospital of Bengbu Medical College). After the initial screening, we found 76 patients that met the criteria for study. Of the 76 patients, 23 cases were found with closed ultrasound window via the TCD examination, 3 cases were found to be microemboli-positive patients the first time but refused to accept the second microemboli detection, and 1 patient failed to follow-up 3 months later. Ultimately, 49 patients within $48 \mathrm{~h}$ of onset of atherosclerosis thrombosis cerebral infarction were included in the study.

\section{Risk factors for stroke patients who initially scored microemboli-positive and negative and comparisons of general information}

Statistical analyses showed that coronary heart disease history $(\mathrm{OR}=5.167,95 \% \mathrm{CI}$ $=1.044-25.565, \mathrm{P}=0.047)$ and the history of antithrombotic therapy $(\mathrm{OR}=0.108,95 \% \mathrm{CI}=$ $0.021-0554, \mathrm{P}=0.003$, protective factors) were significantly different, as was the history of stroke $(\mathrm{OR}=5.818,95 \% \mathrm{CI}=0.933-36.284, \mathrm{P}=0.062)$, the history of hypertension $(\mathrm{OR}=$ $4.550,95 \% \mathrm{CI}=0.884-23.407, \mathrm{P}=0.053)$, and smoking history $(\mathrm{OR}=2.750,95 \% \mathrm{CI}=0.788$ $9.596, \mathrm{P}=0.098)$. There were no significant differences between gender, age, total cholesterol, hematocrit, C-reactive protein, or fibrinogen (Table 1).

\section{A comparison of intracranial and extracranial artery stenosis and microemboli incidence}

With TCD, carotid ultrasound, and MRA, we examined patients on the neck, intracranial carotid artery (CCA), internal carotid artery (ICA), middle cerebral artery (MCA), anterior cerebral artery (ACA), vertebral artery (VA), basilar artery (BA), and posterior cerebral artery (PCA). Of the 49 patients, 123 main artery atherosclerotic stenosis or plaques formed, 33 patients had symptomatic stenosis and 16 patients had asymptomatic stenosis; patients with symptomatic stenosis $(\mathrm{OR}=6.778,95 \% \mathrm{CI}=0.977-47.183, \mathrm{P}=0.009)$ have a higher incidence of microemboli than patients with asymptomatic stenosis $(\mathrm{P}=0.009)$. The incidence of stenosis or plaque formation in the common carotid artery $(\mathrm{OR}=7.000,95 \% \mathrm{CI}=1.800-27.222, \mathrm{P}=0.005)$, internal carotid artery $(\mathrm{OR}=6.500,95 \% \mathrm{CI}=1.712-24.683, \mathrm{P}=0.005)$, and middle cerebral artery $(\mathrm{OR}=$ $4.408,95 \% \mathrm{CI}=1.188-16.362, \mathrm{P}=0.027$ ) in patients with microemboli increased (Table 2 ). 
Table1. Risk factors for the patients with stroke who showed microembolus-positive and negative for the first time and the comparisons of general information.

\begin{tabular}{|c|c|c|c|c|}
\hline \multirow[t]{2}{*}{ Terms } & \multirow[t]{2}{*}{ No. of patients $(\mathrm{N})$} & \multicolumn{2}{|c|}{ First microembolus examination } & \multirow[t]{2}{*}{$\mathrm{P}$} \\
\hline & & $\begin{array}{l}\text { Microembolus-positive } \\
\qquad(\mathrm{N}=15)\end{array}$ & $\begin{array}{l}\text { Microembolus-negative } \\
(\mathrm{N}=34)\end{array}$ & \\
\hline Gender (male, \%) & 35 & $10(66.7 \%)$ & $25(71.4 \%)$ & 0.434 \\
\hline Age (means \pm SD, years) & & $63.13 \pm 6.50$ & $63.59 \pm 7.14$ & 0.208 \\
\hline Stroke history $(\mathrm{N}, \%)$ & 6 & $4(26.7 \%)$ & $2(5.9 \%)$ & 0.062 \\
\hline Hypertension history (N, \%) & 33 & $13(86.7 \%)$ & $20(58.8 \%)$ & 0.219 \\
\hline Diabetes history $(\mathrm{N}, \%)$ & 18 & $7(46.7 \%)$ & $111(32.4 \%)$ & 0.260 \\
\hline Coronary heart disease history $(\mathrm{N}, \%)$ & 8 & $5(33.3 \%)$ & $3(8.8 \%)$ & 0.047 \\
\hline Drinking history $(\mathrm{N}, \%)$ & 19 & $6(40.0 \%)$ & $13(38.2 \%)$ & 0.907 \\
\hline Smoking history $(\mathrm{N}, \%)$ & 21 & $9(60.0 \%)$ & $12(35.3 \%)$ & 0.316 \\
\hline Antithrombotic therapy history $(\mathrm{N}, \%)$ & 22 & $2(13.3 \%)$ & $20(58.8 \%)$ & 0.003 \\
\hline Hematocrit (means $\pm \mathrm{SD}, \%$ ) & & $38.32 \pm 3.25$ & $40.17 \pm 5.44$ & 0.107 \\
\hline Platelet counts (means $\pm \mathrm{SD}, \times 10^{9}$ ) & & $215.0 \pm 53.13$ & $229.1 \pm 71.62$ & 0.328 \\
\hline Fibrinogen (means $\pm \mathrm{SD}, \mathrm{mg}$ ) & & $2.893 \pm 0.612$ & $2.824 \pm 0.850$ & 0.384 \\
\hline C-reactive protein (means $\pm \mathrm{SD}, \mathrm{mM})$ & & $2.836 \pm 3.482$ & $3.326 \pm 5.259$ & 0.983 \\
\hline Glucose $($ means $\pm \mathrm{SD}, \mathrm{mM})$ & & $8.100 \pm 3.732$ & $6.740 \pm 3.453$ & 0.280 \\
\hline Total cholesterol (means $\pm \mathrm{SD}, \mathrm{mM})$ & & $4.727 \pm 0.903$ & $4.759 \pm 1.071$ & 0.575 \\
\hline Triglyceride (means $\pm \mathrm{SD}, \mathrm{mM}$ ) & & $1.973 \pm 1.370$ & $1.467 \pm 0.838$ & 0.173 \\
\hline Low density lipoprotein (means $\pm \mathrm{SD}, \mathrm{mM}$ ) & & $2.913 \pm 0.774$ & $2.979 \pm 0.876$ & 0.737 \\
\hline High density lipoprotein (means $\pm \mathrm{SD}, \mathrm{mM}$ ) & & $1.132 \pm 0.273$ & $1.116 \pm 0.275$ & 0.444 \\
\hline Apolipoprotein $\mathrm{A}$ (means $\pm \mathrm{SD}, \mathrm{mM})$ & & $1.153 \pm 0.229$ & $1.182 \pm 0.232$ & 0.955 \\
\hline Apolipoprotein $\mathrm{B}$ (means $\pm \mathrm{SD}, \mathrm{mM})$ & & $0.980 \pm 0.227$ & $1.043 \pm 0.269$ & 0.579 \\
\hline Homocysteine (means $\pm \mathrm{SD}, \mathrm{mM})$ & & $12.80 \pm 4.89$ & $16.26 \pm 7.14$ & 0.315 \\
\hline Glutamyltranspeptidase (means $\pm \mathrm{SD}, \mu / \mathrm{L}$ ) & & $35.46 \pm 11.84$ & $42.19 \pm 36.68$ & 0.196 \\
\hline
\end{tabular}

Table 2. Incidence comparison of intracranial and extra-cranial artery stenosis and microemboli.

\begin{tabular}{|c|c|c|c|c|}
\hline \multirow[t]{2}{*}{ Terms } & \multirow[t]{2}{*}{ No. of patients $(\mathrm{N})$} & \multicolumn{2}{|c|}{ First microembolus examination } & \multirow[t]{2}{*}{$\mathrm{P}$} \\
\hline & & $\begin{array}{l}\text { Microembolus-positive } \\
\qquad(\mathrm{N}=15)\end{array}$ & $\begin{array}{l}\text { Microembolus-negative } \\
\qquad(\mathrm{N}=34)\end{array}$ & \\
\hline \multicolumn{5}{|l|}{ Symptom status } \\
\hline Symptomatic & 33 & $14(93.3 \%)$ & $19(55.9 \%)$ & 0.009 \\
\hline \multicolumn{5}{|l|}{ Stenosis } \\
\hline Asymptomatic & 16 & $1(6.7 \%)$ & $15(44.1 \%)$ & \\
\hline Common carotid artery stenosis or plaque & 15 & $9(60.0 \%)$ & $6(17.6 \%)$ & 0.005 \\
\hline Internal carotid artery stenosis or plaque & 18 & $10(66.7 \%)$ & $8(23.5 \%)$ & 0.005 \\
\hline Middle cerebral artery stenosis or plaque & 15 & $8(53.3 \%)$ & $7(20.6 \%)$ & 0.027 \\
\hline Anterior cerebral artery stenosis or plaque & 20 & $7(46.7 \%)$ & $13(38.2 \%)$ & 0.403 \\
\hline Vertebral artery stenosis or plaque & 16 & $8(53.3 \%)$ & $13(38.2 \%)$ & 0.325 \\
\hline Basilar artery stenosis or plaque & 11 & $3(20.0 \%)$ & $7(20.6 \%)$ & 0.642 \\
\hline Posterior cerebral artery stenosis or plaque & 13 & $4(26.7 \%)$ & $8(23.5 \%)$ & 0.540 \\
\hline
\end{tabular}

\section{A plaque status examination with carotid artery ultrasound and a comparison of microemboli incidence}

Statistical analyses showed that the thickness of the intima was statistically associated with the incidence of microemboli and patients with unstable plaques had a higher microembolus-positive rate $(\mathrm{P}=0.001$, Table 3$)$.

\section{Effect of microemboli on NIHSS score of neurological impairment}

We scored the NIHSS neurological impairment of patients at the beginning of hos- 
pitalization and a week after admission. We compared scores before and after admission and divided the patients into two groups: improvement or no change and progress. The results showed that patients who were microembolus-positive and negative in the first microembolus examination at the beginning of admission were not statistically different in NIHSS score or NIHSS severity $(\mathrm{P}=0.134, \mathrm{P}=0.351)$. A week after the admission, the re-evaluation of NIHSS scores showed changes that were significantly different $(P=0.026$, Table 4$)$.

Table 3. Plaque status examination with carotid artery ultrasound and the comparison of microemboli incidence.

\begin{tabular}{|c|c|c|c|c|}
\hline \multirow[t]{2}{*}{ Terms } & \multirow[t]{2}{*}{ No. of patients $(\mathrm{N})$} & \multicolumn{2}{|c|}{ First microembolus examination } & \multirow[t]{2}{*}{$P$} \\
\hline & & $\begin{array}{l}\text { Microembolus-positive } \\
(\mathrm{N}=15)\end{array}$ & $\begin{array}{c}\text { Microembolus-negative } \\
(\mathrm{N}=34)\end{array}$ & \\
\hline \multicolumn{2}{|c|}{ Left common carotid artery (means $\pm \mathrm{SD}, \mathrm{mm}$ ) } & $11.00 \pm 2.48$ & $9.71 \pm 1.84$ & 0.038 \\
\hline \multicolumn{2}{|c|}{ Right common carotid artery (means $\pm \mathrm{SD}, \mathrm{mm}$ ) } & $11.73 \pm 3.62$ & $9.24 \pm 1.76$ & 0.041 \\
\hline \multicolumn{2}{|c|}{ Left internal carotid artery (means $\pm \mathrm{SD}, \mathrm{mm})$} & $9.80 \pm 3.03$ & $8.24 \pm 1.79$ & 0.037 \\
\hline \multicolumn{2}{|c|}{ Right internal carotid artery (means $\pm \mathrm{SD}, \mathrm{mm}$ ) } & $9.07 \pm 3.08$ & $8.59 \pm 1.96$ & 0.007 \\
\hline \multicolumn{5}{|c|}{ Carotid artery ultrasound } \\
\hline \multicolumn{5}{|l|}{ Plaques status } \\
\hline No plaques & 16 & $3(20.0 \%)$ & $3(38.2 \%)$ & \multirow[t]{3}{*}{0.001} \\
\hline Stable plaques & 21 & $3(20.0 \%)$ & $18(52.9 \%)$ & \\
\hline Unstable plaques & 12 & $9(60.0 \%)$ & $3(8.8 \%)$ & \\
\hline
\end{tabular}

Table 4. Effect of microemboli on NIHSS score of neurological impairment.

\begin{tabular}{lccc}
\hline Terms & No. of patients $(\mathrm{N})$ & \multicolumn{2}{c}{ First microembolus examination } \\
\cline { 3 - 4 } & & $\begin{array}{c}\text { Microembolus-positive } \\
(\mathrm{N}=15)\end{array}$ & $\begin{array}{c}\text { Microembolus-negative } \\
(\mathrm{N}=34)\end{array}$ \\
\hline NIHSS score (means \pm SD, points) & & $6.40 \pm 3.98$ & $4.38 \pm 3.10$ \\
Stroke severity (mild to moderate, \%) & 36 & $10(66.7 \%)$ & $26(76.5 \%)$ \\
NIHSS Score changes & 34 & $6(66.7 \%)$ & 0.134 \\
$\quad$ Improvement or no change & 5 & $3(33.3 \%)$ & $38(95.0 \%)$ \\
Progress & & $2(5.0 \%)$ & 0.351 \\
\hline
\end{tabular}

We retested 15 cases of patients with positive microembolus detection a week later, in which 9 cases were positive. They showed a significant difference $(P=0.036$, Table 5$)$ in NIHSS score changes with the remaining 40 cases of patients.

\begin{tabular}{|c|c|c|c|c|}
\hline \multirow[t]{2}{*}{ Terms } & \multirow[t]{2}{*}{ No. of patients $(\mathrm{N})$} & \multicolumn{2}{|c|}{ Second microembolus examination } & \multirow[t]{2}{*}{$\mathrm{P}$} \\
\hline & & Microembolus-positive $(\mathrm{N}=9)$ & Microembolus-negative $(\mathrm{N}=40)$ & \\
\hline \multicolumn{5}{|l|}{ NIHSS Score changes } \\
\hline Improvement or no change & 34 & $6(66.7 \%)$ & $38(95.0 \%)$ & 0.037 \\
\hline Progress & 5 & $3(33.3 \%)$ & $2(5.0 \%)$ & \\
\hline
\end{tabular}

\section{Relationship between microemboli and the mRS score changes within three months}

We performed mRS scoring for all patients before discharge. Three months later, we followed up 36 patients directly and with another 13 patients through telephone and performed 
mRS scoring. There were 4 cases of death and 3 cases of recurrence that were considered as deterioration during hospitalization and the 3-month follow-up period. Statistical analyses showed that there were no significant changes between $\mathrm{mRS}$ score changes and the positivity rate at the first microembolus examination $(\mathrm{P}=0.319$, Table 6).

\begin{tabular}{|c|c|c|c|c|}
\hline \multirow[t]{2}{*}{ Terms } & \multirow[t]{2}{*}{ No. of patients $(\mathrm{N})$} & \multicolumn{2}{|c|}{ First microembolus examination } & \multirow[t]{2}{*}{$\mathrm{P}$} \\
\hline & & Microembolus-positive $(\mathrm{N}=15)$ & Microembolus-negative $(\mathrm{N}=34)$ & \\
\hline \multicolumn{5}{|l|}{ mRS changes } \\
\hline Improved & 26 & $7(46.7 \%)$ & $19(55.9 \%)$ & 0.319 \\
\hline No changes & 13 & $3(20.0 \%)$ & $10(19.4 \%)$ & \\
\hline Deterioration & 10 & $5(33.3 \%)$ & $5(14.7 \%)$ & \\
\hline
\end{tabular}

\section{Relationships between microemboli and stroke recurrence and endpoint events}

Of the 49 patients, there were 3 cases of recurrence: 1 microembolus-positive patient within one week, 1 patient who was microembolus-negative within 3 months, and 1 patient who was microembolus-positive within 3 months. There were four cases of death: 2 patients who were microembolus-positive within one week, 1 patient who was microembolus-negative, and 1 patient who was microembolus-negative within 3 months. The endpoint events (including recurrence and death) of patients who were microembolus-positive and -negative did not show a significant difference $(P=0.179$, Table 7$)$.

Table 7. Relationships between microemboli and stroke recurrence and endpoint events within 3 months.

\begin{tabular}{lcccr}
\hline Terms & No. of patients (N) & \multicolumn{2}{c}{ First microembolus examination } & $\mathrm{P}$ \\
\cline { 3 - 4 } & & Microembolus-positive $(\mathrm{N}=15)$ & Microembolus-negative (N=34) \\
\hline Stroke recurrence & 3 & $2(13.3 \%)$ & $1(2.9 \%)$ & 0.218 \\
Stroke death & 4 & $2(13.3 \%)$ & $2(5.9 \%)$ & 0.576 \\
Endpoint events (death or recurrence) & 7 & $4(26.7 \%)$ & $3(8.8 \%)$ & 0.179 \\
\hline
\end{tabular}

\section{DISCUSSION}

In three years, we ultimately collected 49 confirmed cases of patients with atherosclerotic cerebral infarction (referred to as the main artery of cerebral infarction). The frequency of patients who were microembolus-positive within $48 \mathrm{~h}$ of stroke at the first examination was $30.6 \%$. The incidence rate $60.0 \%$ after one week of stroke, and the total positive incidence rate is $18.4 \%$. For the third examination three months later, all patients were negative by microembolus examination. Iguchi et al. (2007) studied 143 cases of patients with atherosclerotic cerebral infarction and determined the ratio of patients who were microembolus-positive within 24 $h$ of onset. These authors found that $43 \%$ of the patients were again detected as microemboluspositive one week later, and the total frequency of microembolus-positive patients in one week was $7 \%$, which is close to our conclusion. Studies have shown that the ratio of microemboluspositivity peaks immediately after stroke, and the ratio decreases as time goes on (Sliwka et al., 1997; Iguchi et al., 2007; Hao et al., 2010).

Through medical analysis of the groups of patients, we discovered that a history of 
coronary heart disease $(\mathrm{OR}=5.167, \mathrm{P}=0.047)$ or antithrombotic therapy $(\mathrm{OR}=0.108, \mathrm{P}=$ 0.003 ) significantly affected the ratio of microemboli. We believe that coronary heart disease has a similar etiology and risk factors with artery cerebral infarction, and the presence of coronary atherosclerosis indicated that the incidence of atherosclerosis increases in the neck and head. Studies have shown that antithrombotic treatments, such as antiplatelet, anticoagulant, and defibrinogen, could reduce the ratio of the detection of the first microemboli after stroke (Goertler et al., 1999). With registered large-scale clinical studies, such as the CARESS test (Markus and MacKinnon, 2005) and CLAIR test, we studied the effect of a clopidogrel and aspirin combination treatment on the prognosis of microembolus-positive patients. The results showed that the combination of clopidogrel and aspirin could better decrease the chance of microembolus detection in microembolus-positive patients than aspirin alone.

Through carotid artery ultrasound plaque examination of the patients in the study, 9 of the 12 patients with soft plaque, ulcerative plaque, and other unstable plaques were detected as positive in a microembolus examination, and the ratio of microembolus-positive detection (detection ratio $=0.75)$ was significantly higher than in patients with stable or no plaques $(\mathrm{P}=0.001)$, which was also consistent with previous studies. We believe that the increase in the microemboluspositivity ratio in patients with unstable plaques was associated with the composition and source of microemboli. Microembolus signals detected with TCD include blood clots, platelet aggregated particles, arteriosclerosis plaque particles (platelets, fibrinogen, cholesterol), and fat or air. However, the majority of microemboli from artery cerebral infarction were particles enriched in platelets that were caused by the rupture of atherosclerosic unstable plaques (Goertler et al., 2002; Junghans and Siebler, 2003). Sitzer et al. (1995) found that atherosclerotic plaques and intraluminal thrombus from carotid ulcerations were the main source of microemboli.

In the 15 patients who were microemboli-positive, 14 patients had symptomatic stenosis, suggesting that patients with symptomatic stenosis are more prone to be detected as microembolus-positive $(\mathrm{P}=0.009)$. We also found that the incidence of microemboli was associated with the stenosis of the carotid artery, internal carotid artery and middle cerebral artery, whereas the stenosis of anterior cerebral artery and back circulatory system had no significant effect on the incidence of microemboli, which is similar to previous conclusions (Ritter, 2008; Zhang et al., 2008; Wisselink, 2009). We speculate that these observations may be associated with the operations during microembolus examination with TCD. Probes were placed on the temporal window of corresponding vessels to detect the blood flow of the middle cerebral artery, thus the microemboli from corresponding lesion vessels in the common carotid artery, internal carotid artery, and middle cerebral artery were more easily detected.

Studies showed that the incidence of microemboli increased with the increased degree of stenosis. Further studies by Del Sette et al. (1997) showed that the microembolus signals could not be detected when the carotid stenosis was $<50 \%$. A carotid stenosis stratified study found that carotid stenosis was $<50 \%$. In addition, the frequency of microembolus-positivity reached $10 \%$ and $22 \%$ when carotid stenosis reached $50-70 \%$ or $>75 \%$, respectively. The microembolus-positive frequency reached 39\% among patients with carotid occlusion. Gao et al. (2004) studied intracranial middle cerebral artery stenosis and found that the microemboluspositive frequency was $48 \%$ (10/21 cases) in patients with severe stenosis, and the frequency was $15 \%$ (4/26 cases) in patients with moderate stenosis.

The study found that the existence of microemboli may increase the NIHSS score in one week and aggravate neural impairment $(\mathrm{P}=0.026, \mathrm{P}=0.036)$, suggesting that progres- 
sive strokes were more likely to occur within one week in microembolus-positive patients, which was different from what was observed in previous studies (Lund et al., 2000; Abbott et al., 2005). The mRS score did not show statistical differences just before and 3 months after discharge $(\mathrm{P}=0.319)$, suggesting that the presence of microemboli had no significant effect on the improvement of neurological function after 3 months. Hao et al. (2010) found that the NIHSS score improved more significantly in patients with microemboli and turned negative in patients with continuously positive detection of microemboli. Iosif et al. (2008) and Schlaug et al. (1999) re-examined MRI DWI and perfusion-weighted imaging (PWI) on patients within 1 week after stroke onset and found that asymptomatic cerebral perfusion was more likely to decrease in microembolus-positive patients and that small infarct lesions that could not be detected with MRI DWI also emerged.

Progressive stroke is caused by multiple factors. Studies have shown that antithrombotic therapy can help reduce the incidence of progressive stroke. Neural function improved more significantly in patients that became microembolus-negative than in continuously microembolus-positive patients (Iguchi et al., 2008). We speculate that antithrombotic therapy, in particular, the combination of clopidogrel and aspirin, could reduce the incidence of progressive stroke, which is partially because of the clearance of microemboli in the cerebral blood, which reduces the incidence of microemboli. We further speculate that microemboluspositivity may be a predictor of cerebral infarction progression.

Previous studies have shown that the presence of microemboli increases the probability of stroke relapse. Iguchi et al. (2008) also discovered that $48 \mathrm{~h}$ after stroke, but not within 24 $\mathrm{h}$, microembolus-positivity was associated with stroke recurrence. Therefore, we performed microembolus detection $48 \mathrm{~h}$ after stroke for the first time. In our study, there were 2 cases of microembolus-positive recurrence, with a rate of $13.3 \%(2 / 15)$. There was 1 case of microembolus-negative recurrence with a rate of $2.9 \%(1 / 34)$. The recurrence rate was higher in microembolus-positive patients than in microembolus-negative patients, but was not significantly different $(\mathrm{P}=0.218)$, which may be due to the small sample size and low total recurrence rate. Furthermore, patients without new symptoms of stroke were not reexamined for MRI DWI. Kang et al. (2007) have shown that $34 \%$ of patients could develop a new micro-infarction without obvious clinical symptoms and without significant NIHSS score changes in one week.

Abbott et al. (2005) have shown that endpoint events, like stroke recurrence and poststroke death, were more likely to occur in microembolus-positive than in microembolus-negative patients. Cerebral infarction recurrence (three cases) or deaths (four cases) directly caused by cerebral infarction during hospitalization and discharge within 3 months were considered to be endpoint events. In addition, we found that the rate of endpoint events was higher in microembolus-positive patients ( 2 cases, with a mortality rate of $2 / 15(13.3 \%)$ within $48 \mathrm{~h}$ of cerebral infarction onset than in microembolus-negative patients [ 2 cases, with a mortality rate of $2 / 34(5.9 \%)$ ] but was not significantly different $(\mathrm{P}=0.178)$. We speculate that this may be associated with the short follow-up period and small sample size. Therefore, in order to prevent the progress and recurrence of stroke in cerebral infarction patients and decrease the mortality rate of stroke, it is necessary to carry out a study with a larger sample size and more in-depth study of microemboli.

\section{REFERENCES}

Abbott AL, Chambers BR, Stork JL, Levi CR, et al. (2005). Embolic signals and prediction of ipsilateral stroke or transient 
ischemic attack in asymptomatic carotid stenosis: a multicenter prospective cohort study. Stroke 36: 1128-1133.

Ackerstaff R, Babikian V, Georgiadis D, Russell D, et al. (1995). Basic identification criteria of Doppler microembolic signals. Consensus Committee of the Ninth International Cerebral Hemodynamic Symposium. Stroke 26: 1123.

DeGraba TJ, Hallenbeck JM, Pettigrew KD, Dutka AJ, et al. (1999). Progression in acute stroke: value of the initial NIH stroke scale score on patient stratification in future trials. Stroke 30: 1208-1212.

Del Sette M, Angeli S, Stara I, Finocchi C, et al. (1997). Microembolic signals with serial transcranial Doppler monitoring in acute focal ischemic deficit. A local phenomenon? Stroke 28: 1311-1313.

Gao S, Wong KS, Hansberg T, Lam WW, et al. (2004). Microembolic signal predicts recurrent cerebral ischemic events in acute stroke patients with middle cerebral artery stenosis. Stroke 35: 2832-2836.

Goertler M, Baeumer M, Kross R, Blaser T, et al. (1999). Rapid decline of cerebral microemboli of arterial origin after intravenous acetylsalicylic acid. Stroke 30: 66-69.

Goertler M, Blaser T, Krueger S, Hofmann K, et al. (2002). Cessation of embolic signals after antithrombotic prevention is related to reduced risk of recurrent arterioembolic transient ischaemic attack and stroke. J. Neurol. Neurosurg. Psychiatry 72: 338-342.

Goldstein LB, Jones MR, Matchar DB, Edwards LJ, et al. (2001). Improving the reliability of stroke subgroup classification using the Trial of ORG 10172 in Acute Stroke Treatment (TOAST) criteria. Stroke 32: 1091-1098.

Hao Q, Leung WH, Leung C, Mok CT, et al. (2010). The significance of microembolic signals and new cerebral infarcts on the progression of neurological deficit in acute stroke patients with large artery stenosis. Cerebrovasc. Dis. 29: 424-430.

Iguchi Y, Kimura K, Kobayashi K, Yamashita S, et al. (2007). Microembolic signals after 7 days but not within 24 hours of stroke onset should be predictor of stroke recurrence. J. Neurol. Sci. 263: 54-58.

Iguchi Y, Kimura K, Kobayashi K, Ueno Y, et al. (2008). Microembolic signals at 48 hours after stroke onset contribute to new ischaemia within a week. J. Neurol. Neurosurg. Psychiatry 79: 253-259.

Iosif C, Oppenheim C, Lamy C, Mas JL, et al. (2008). Bilateral hypoperfusion and normal diffusion MR images in a case of acute embolic stroke. Cerebrovasc. Dis. 26: 95-96.

Junghans U and Siebler M (2003). Cerebral microembolism is blocked by tirofiban, a selective nonpeptide platelet glycoprotein IIb/IIIa receptor antagonist. Circulation 107: 2717-2721.

Kang DW, Kwon SU, Yoo SH, Kwon KY, et al. (2007). Early recurrent ischemic lesions on diffusion-weighted imaging in symptomatic intracranial atherosclerosis. Arch. Neurol. 64: 50-54.

Kimura K, Minematsu K, Koga M, Arakawa R, et al. (2001). Microembolic signals and diffusion-weighted MR imaging abnormalities in acute ischemic stroke. AJNR Am. J. Neuroradiol. 22: 1037-1042.

Lund C, Rygh J, Stensrod B, Sandset PM, et al. (2000). Cerebral microembolus detection in an unselected acute ischemic stroke population. Cerebrovasc. Dis. 10: 403-408.

Markus HS, Droste DW, Kaps M, Larrue V, et al. (2005). Dual antiplatelet therapy with clopidogrel and aspirin in symptomatic carotid stenosis evaluated using doppler embolic signal detection: the Clopidogrel and Aspirin for Reduction of Emboli in Symptomatic Carotid Stenosis (CARESS) trial. Circulation 111: 2233-2240.

Markus HS and MacKinnon A (2005). Asymptomatic embolization detected by Doppler ultrasound predicts stroke risk in symptomatic carotid artery stenosis. Stroke 36: 971-975.

Nakajima M, Kimura K, Shimode A, Miyashita F, et al. (2007). Microembolic signals within 24 hours of stroke onset and diffusion-weighted MRI abnormalities. Cerebrovasc. Dis. 23: 282-288.

Ritter MA, Dittrich R, Thoenissen N, Ringelstein EB, et al. (2008). Prevalence and prognostic impact of microembolic signals in arterial sources of embolism. A systematic review of the literature. J. Neurol. 255: 953-961.

Schlaug G, Benfield A, Baird AE, Siewert B, et al. (1999). The ischemic penumbra: operationally defined by diffusion and perfusion MRI. Neurology 53: 1528-1537.

Sitzer M, Müller W, Siebler M, Hort W, et al. (1995). Plaque ulceration and lumen thrombus are the main sources of cerebral microemboli in high-grade internal carotid artery stenosis. Stroke 26: 1231-1233.

Sliwka U, Lingnau A, Stohlmann WD, Schmidt P, et al. (1997). Prevalence and time course of microembolic signals in patients with acute stroke. A prospective study. Stroke 28: 358-363.

Spencer MP, Thomas GI, Nicholls SC and Sauvage LR (1990). Detection of middle cerebral artery emboli during carotid endarterectomy using transcranial Doppler ultrasonography. Stroke 21: 415-423.

Valton L, Larrue V, le Traon AP, Massabuau P, et al. (1998). Microembolic signals and risk of early recurrence in patients with stroke or transient ischemic attack. Stroke 29: 2125-2128.

Wisselink W (2009). Determinants of microembolic signals in patients with atherosclerotic plaques of the internal carotid artery. Eur. J. Vasc. Endovasc. Surg. 38: 148.

Wong KS, Gao S, Chan YL, Hansberg T, et al. (2002). Mechanisms of acute cerebral infarctions in patients with middle cerebral artery stenosis: a diffusion-weighted imaging and microemboli monitoring study. Ann. Neurol. 52: 74-81.

Genetics and Molecular Research 13 (3): 6734-6745 (2014) 
Zhang CM, Wu CJ and Qu SB (2008). Study of Microembolic signal in patients with asymptomatic carotid stenosis by ultrasound. Chin. J. Ultrasonography 17: 112-117. 